\title{
The effect of social information from live demonstrators compared to video playback on blue tit foraging decisions
}

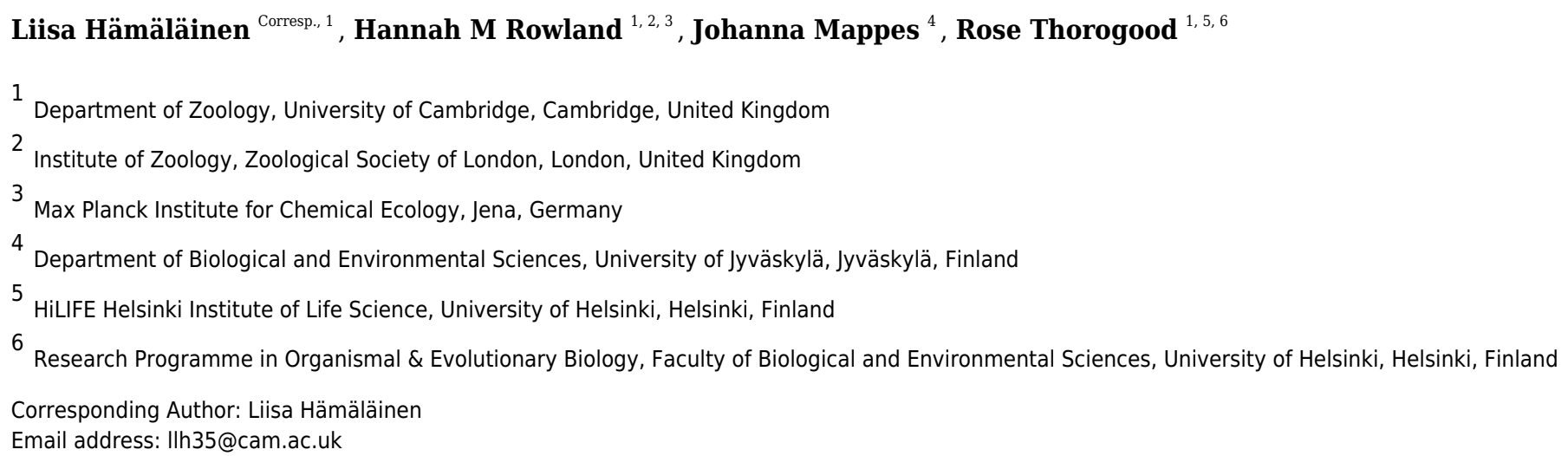

Video playback provides a promising method to study social interactions, and the number of video playback experiments has been growing in recent years. Using videos has advantages over live individuals as it increases the repeatability of demonstrations, and enables researchers to manipulate the features of the presented stimulus. How observers respond to video playback might, however, differ among species, and the efficacy of video playback should be validated by investigating if individuals' responses to videos are comparable to their responses to live demonstrators. Here we use a novel foraging task to compare blue tits' (Cyanistes caeruleus) responses to social information from a live conspecific versus video playback. Birds first received social information about the location of food, and were then presented with a three-choice foraging task where they could search for food from locations marked with different symbols (cross, square, plain white). Two control groups saw only a foraging tray with similar symbols but no information about the location of food. We predicted that socially educated birds would prefer the same location where a demonstrator had foraged, but we found no evidence that birds copied a demonstrator's choice, regardless of how social information was presented. Social information, however, had an influence on blue tits' foraging choices, as socially educated birds seemed to form a stronger preference for a square symbol (against two other options, cross and plain white) than the control birds. Our results suggest that blue tits respond to video playback of a conspecific similarly as to a live bird, but how they use this social information in their foraging decisions, remains unclear. 
1 The effect of social information from live demonstrators compared to video

2 playback on blue tit foraging decisions

3

4 Liisa Hämäläinen ${ }^{1}$, Hannah M. Rowland ${ }^{1,2,3}$, Johanna $\operatorname{Mappes}^{4}$, \& $\operatorname{Rose~Thorogood~}^{1,5,6}$

5

$6 \quad{ }^{1}$ Department of Zoology, University of Cambridge, UK

$7 \quad{ }^{2}$ Institute of Zoology, Zoological Society of London, UK

$8 \quad{ }^{3}$ Max Planck Institute for Chemical Ecology, Jena, Germany

$9{ }^{4}$ Department of Biological and Environmental Sciences, University of Jyväskylä, Finland

$10{ }^{5}$ HiLIFE Helsinki Institute of Life Science, University of Helsinki, Finland

$11{ }^{6}$ Research Programme in Organismal \& Evolutionary Biology, Faculty of Biological and

12 Environmental Sciences, University of Helsinki, Finland

\section{Correspondence:}

Liisa Hämäläinen

Department of Zoology, University of Cambridge,

Downing Str, Cambridge, CB2 3EJ, United Kingdom

Email:11h35@cam.ac.uk 
21 ABSTRACT

22

Video playback provides a promising method to study social interactions, and the number of video playback experiments has been growing in recent years. Using videos has advantages over live individuals as it increases the repeatability of demonstrations, and enables researchers to manipulate the features of the presented stimulus. How observers respond to video playback might, however, differ among species, and the efficacy of video playback should be validated by investigating if individuals' responses to videos are comparable to their responses to live demonstrators. Here we use a novel foraging task to compare blue tits' (Cyanistes caeruleus) responses to social information from a live conspecific versus video playback. Birds first received social information about the location of food, and were then presented with a three-choice foraging task where they could search for food from locations marked with different symbols (cross, square, plain white). Two control groups saw only a foraging tray with similar symbols but no information about the location of food. We predicted that socially educated birds would prefer the same location where a demonstrator had foraged, but we found no evidence that birds copied a demonstrator's choice, regardless of how social information was presented. Social information, however, had an influence on blue tits' foraging choices, as socially educated birds seemed to form a stronger preference for a square symbol (against two other options, cross and plain white) than the control birds. Our results suggest that blue tits respond to video playback of a conspecific similarly as to a live bird, but how they use this social information in their foraging decisions, remains unclear. 


\section{INTRODUCTION}

47 The number of studies investigating social information use in animals has been expanding during 48 the last few decades, and it is now well documented that many species use social information in 49 their decision-making (Galef \& Laland, 2005). Acquiring social information can be beneficial in many different contexts. Animals can, for example, use social information in their foraging

51 decisions, mate choice, breeding habitat selection, or when avoiding predators (Danchin et al., 2004). Social transmission is taxonomically widespread, with evidence of social information use found in birds (Aplin, 2019), mammals (Whiten, 2000), fish (Brown \& Laland, 2003), reptiles

(Noble, Byrne \& Whiting, 2014; Kis, Huber \& Wilkinson, 2015) and insects (Dawson \& Chittka, 2012; Baracchi et al., 2018). Social information is predicted to benefit individuals by reducing the costs of personal learning (Laland, 2004; Kendal et al., 2005; Kendal et al., 2018). When foraging, for example, individuals can gather social information about the location of food sources or food palatability, and learn novel foraging skills (reviewed in Galef \& Giraldeau, 2001), which could increase their foraging efficiency.

As the number of social learning studies has grown, also the number of techniques to study social interactions has increased. A common method is to use artificial stimuli that enables researchers to control and standardize what information is presented (D'Eath, 1998; Woo \& Rieucau, 2011). Artificial stimuli have been used for a long time in animal behaviour research, starting from simple dummies and leading up to robotic animals. Cardboard models were first used by Tinbergen $\&$ Perdeck (1950) to investigate the importance of various stimulus characteristics 
67 on the begging response of herring gull chicks. Subsequently, simple models have been used in

68

69 many experiments, including studies investigating mate choice (Halnes \& Gould, 1994; Höglund et al., 1995), or individuals' responses to predators (Powell, 1974; Petersson \& Järvi, 2006) and brood parasites (Thorogood \& Davies, 2016). Over the recent years, new technology has enabled researchers to use also more sophisticated techniques, such as robotic animals (Taylor et al., 2008; Krause, Winfield \& Deneubourg, 2011). For example, male satin bowerbirds were found to adjust their displays in response to signals from robotic females (Patricelli et al., 2002), and wild grey squirrels were shown to respond to a robotic model of a conspecific displaying alarm behaviour (Partan, Larco \& Owens, 2009).

Another promising technique to study social interactions is video playback. Videos can be easily edited and manipulated, allowing researchers to alter the stimulus features that are presented to observers and reduce the variation among presentations (D'Eath, 1998). Video presentations can be used to study animals' responses to simple animations, such as point-light displays, and domestic chicks (Gallus gallus domesticus) have been demonstrated to prefer biological motion patterns when exposed to these displays (Vallortigara, Regolin \& Marconato, 2005; Vallortigara \& Regolin, 2006). Furthermore, with technological advances it is now possible to create realistic computer-generated animations of animal models to study social interactions (Woo \& Rieucau, 2011). However, a more common method in behavioural studies is to record a video of a live animal and video playback has now been used successfully in many bird species (Adret, 1997; Ikebuchi \& Okanoya, 1999; Ophir \& Galef, 2003; Bird \& Emery, 2008; Rieucau \& Giraldeau, 2009; Guillette \& Healy, 2017; Thorogood, Kokko \& Mappes, 2018; Carouso-Peck \& Goldstein, 2019; Smit \& Oers, 2019), as well as across a range of other taxa, including mammals (Hopper, 
90 Lambeth \& Schapiro, 2012; Gunhold, Whiten \& Bugnyar, 2014), fish (Rowland et al., 1995;

91 Trainor \& Basolo, 2000), reptiles (Clark, Macedonia \& Rosenthal, 1997; Ord et al., 2002) and

92 spiders (Clark \& Uetz, 1992). Video playback does, however, have limitations such as the lack of

93 depth cues, the lack of interaction between an observer and an individual on the video, and

94 differences between animal and human visual systems (D’Eath, 1998; Zeil, 2000; Ware, Saunders

$95 \&$ Troje, 2015). Birds, for example, have higher critical flicker-fusion frequencies $(>100 \mathrm{~Hz})$ than

96 humans $(60 \mathrm{~Hz})$ and they might therefore perceive the video image as flickering, instead of

97 continuous motion (D’Eath, 1998; Bird \& Emery, 2008). However, this degree of visual resolution

98 often occurs when light stimuli are very bright (e.g. $1500 \mathrm{~cd} / \mathrm{m}^{2}$ in blue tits, (Boström et al., 2016))

99 and beyond the normal brightness of most video screens. Furthermore, the use of liquid crystal

100 display (LCD) monitors instead of older cathode ray tube (CRT) displays can help to overcome

101 the problem of flicker, and especially a flickerless thin film transistor (TFT) LCD has provided a

102 good method to present videos to birds (Ikebuchi \& Okanoya, 1999). Another important aspect to

103 take into account is image presentation rate (IPR) which influences how realistic the motion on

104 the video appears (Ware, Saunders \& Troje, 2015). Ware, Saunders \& Troje (2015) demonstrated

105 that pigeons (Columbia livia) responded to videos of a conspecific more strongly when IPR was

10660 frames per second, compared to lower presentation rates (15 or 30 frames/s), and the authors

107 therefore suggest researchers to use the highest frame rate available when using video playback.

Although videos have been used successfully in many studies, video playback does not 110 always generate the same responses in observers when compared to studies using live 111 demonstrators (see Schlupp, 2000). For example, a recent study with California scrub-jays 112 (Aphelocoma californica) found that observing a video of a conspecific eavesdropping on a 
113 caching event did not influence focal individuals' caching and re-caching behaviour, in contrast to

114 previous studies with a live conspecific (Brecht et al., 2018). The strength of the responses to

115 video and live demonstrations may also differ even when observers are found to respond to videos.

116 Zebra finch (Taenopygia guttata) males, for example, copy the nest material choice from a video

117 demonstrator but this preference is stronger when birds observe a live demonstrator (Guillette \&

118 Healy, 2019). Most of these studies, however, have compared individuals' responses to video

119 playback to previous experiments with live demonstrators, and therefore have not accounted for

120 possible differences in test conditions, such as individual differences among the demonstrators.

121 Here our aim was to compare these two methods in one study by investigating whether blue tits'

122 response to the same demonstrator differs between video and live presentation.

The applicability of video playback in studies with blue tits is so far unclear. We found conspecific, but social information from videos did not influence their foraging decisions in a later 127 foraging task (Hämäläinen et al., 2017). In contrast, great tits (Parus major) have been demonstrated to respond to videos of a conspecific (Snijders, Naguib \& van Oers, 2017), and use social information from videos in their foraging decisions (Thorogood, Kokko \& Mappes, 2018; Smit \& Oers, 2019; Hämäläinen et al., 2019), suggesting that video playback can be used successfully in other parid tit species. It is, however, possible that even closely related species differ in their response to video stimuli. For example, Roberts, Gumm \& Mendelson (2017) tested the efficacy of video playback in two species of darters, Etheostoma barrenense and Etheostoma zonale, and found that despite the same experimental set-up and close relatedness of the species, only E. zonale females' responses to video playback of conspecific males were comparable to live 
136 males, whereas E. barrenense females showed a preference only for live males. Similarly, blue tits

137 might respond to videos differently than great tits. Alternatively, our previous result of blue tits

138 not copying a demonstrator (Hämäläinen et al., 2017) might be because blue tits were simply not

139 using acquired social information, regardless of how it was presented. Indeed, studies using live

140 demonstrators have found that only about half of the tested blue tits learn a novel foraging task

141 socially (Sasvári, 1979; Sasvári, 1985; Aplin, Sheldon \& Morand-Ferron, 2013), compared to great

142 tits that are more likely to solve the task after observing others (Sasvári, 1979; Sasvári, 1985). To

143 disentangle the effect of video playback and blue tits' tendency to use social information, we

144 designed an experiment where we investigated whether birds were more likely to use social

145 information from a live demonstrator, compared to a video presentation.

In this experiment, we presented blue tits with a three-choice foraging task: an ice cube tray with three wells covered and marked with different symbols (cross, square and plain white). One group of the birds received social information about the location of food from a live conspecific, whereas another group saw a video playback of a conspecific demonstrator. In addition, we had two control groups that only saw a foraging tray (live/video presentation) but no information about the location of food. We predicted that the birds in the control group would not have a preference for any of the symbols and would choose each of them equally often. Socially educated birds were predicted to choose the same symbol and location where they had observed a demonstrator foraging. We predicted that blue tits would copy a demonstrator's choice equally often regardless of how social information was presented (live/video demonstrator). However, finding that blue tits were less likely to copy a demonstrator's choice from videos would indicate that video playback might not be a suitable method for social learning studies in the species. Finally, we predicted that 
159 birds that received social information would start the foraging task faster than control birds

160 (Hämäläinen et al., 2017; Thorogood, Kokko \& Mappes, 2018).

161

\section{METHODS}

\section{Birds}

164 The experiment was conducted at Konnevesi Research Station in Central Finland during January 165 and February 2017. We tested social information use in 40 juvenile blue tits. In addition, five adult 166 birds were used as demonstrators. Birds were caught from the feeding site and housed in individual 167 plywood cages $(80 \mathrm{~cm}(\mathrm{~h}) \times 65 \mathrm{~cm}(\mathrm{w}) \times 50(\mathrm{~d}) \mathrm{cm})$ with a daily light period of 12.5 hours, and 168 free access to food (sunflower seeds, tallow and peanuts) and fresh water. Before and during the experiment food was restricted to make sure that birds were motivated to forage. Birds were kept

170 in captivity for approximately one week and then released back at the capture site. Before this, each bird was weighted and ringed for identification purposes. The work was carried out with

172 permission from the Central Finland Centre for Economic Development, Transport and 173 Environment and license from the National Animal Experiment Board (ESAVI/9114/ 04.10.07/2014) and the Central Finland Regional Environmental Centre (VARELY/294/2015). Birds were treated following the ASAB guidelines for the treatment of animals in behavioural research and teaching (2012).

\section{Foraging task and pre-training}

179 We investigated whether blue tits used social information about the location of food by presenting them a three-choice foraging task where they had to find mealworms from a white plastic ice cube tray (modifying a protocol used in Hodgson \& Healy, 2005). The tray had 21 wells in three rows 
182 and we covered three of these (in the middle row) with a piece of white paper that had either (i) a

183 black cross symbol, (ii) a black square symbol, or (iii) no symbol (plain white) printed on top (Fig.

184 1a). The same symbols were attached in front of the foraging tray to increase their visibility to the 185 observers during demonstration. In the experiment birds had to lift up the paper covers to find a 186 food reward and we investigated whether social information influenced their first choice.

187

188

189

190

191

192

193

194

195

196

197

198

199

200

201

202

203

204

Before the experiment, we trained birds in their home cages to forage from an ice cube tray.

The training was done step-wise by first offering birds a tray with four of the wells (randomly chosen) containing a mealworm. After birds had eaten these, we next presented them with a tray with four wells partly covered (again, randomly chosen), so that the mealworms were still visible. During the training, we covered the wells with brown paper to prevent birds associating the reward with white colour that was used in the social learning experiment. In the next step birds received a tray where four wells were covered with brown paper, so that the mealworms were completely hidden. After birds had completed these steps (i.e. found and consumed all mealworms), we finally presented them with a tray with seven wells covered but only four of them containing a mealworm. This was done to increase individuals' uncertainty about a food reward in the wells, so that they would be more likely to use social information in the experiment. Training was completed once individuals had found and consumed all mealworms. All birds finished the training in one day.

\section{Demonstrators}

We used five individuals (all adults, i.e. $>1$ year old) as demonstrators in the experiment. Each individual was used twice in the live demonstration and also filmed for the video playback that was presented to two observers (i.e. each individual was demonstrator for four observers). 
205 Demonstrators were first trained to forage from an ice cube tray in their home cages, following a 206 similar step-wise protocol that we used with observers (see above). However, instead of covering 207 the wells with brown paper, we presented demonstrators with a similar tray that we used in the 208 experiment, with three wells covered with different symbols (cross, square, plain white; Fig. 1a).

209 The food reward was placed only under one of the symbols (cross or square) whereas the other 210 wells were always empty. Demonstrators therefore learned to associate a food reward with one of 211 the symbols and searched for food from that location during the demonstrations. We trained two 212 of the demonstrators to associate a food reward with a cross symbol, and two with a square symbol. 213 To ensure that the number of demonstrations for each symbol was balanced, the last of the five 214 demonstrators was trained first with a square and then with a cross. finding a mealworm by lifting up the paper cover) through the plexiglass wall of the test cage (a $66(\mathrm{~h}) \times 50(\mathrm{w}) \times 50(\mathrm{~d}) \mathrm{cm}$ sized plywood cage with the plexiglass front wall) using an HD camcorder Canon Legria HF R66 (with 50 frames/s progressive recording mode). Three mealworms were hidden in the well (with either a cross or a square symbol), and birds were filmed finding and eating all of them, so the demonstration was repeated three times. We then edited these

222 videos (using Windows Movie Maker), so that they were all 150 seconds long (see a video clip in 223 Supplementary material). We also filmed a five-minute long video of a demonstrator in the cage 224 without a tray, which was presented to observers before the foraging task demonstration. Finally, 225 we filmed control videos that contained a tray only (with different symbols) but no bird (150 s). We filmed six different control videos with all possible symbol orders on the tray to ensure that the location on the tray would not influence our results. 


\section{Experimental protocol}

230 In the experiment observers were randomly allocated to four treatments ( $\mathrm{n}=10$ in each): (i) social 231 information from a live demonstrator, (ii) social information from video playback, (iii) live control 232 (the feeding tray only), (iv) video playback control (video of the feeding tray only). In all 233 treatments, birds were first allowed to habituate to the test cage for two hours. During this time, 234 we repeated the foraging task training one more time by presenting birds with an ice cube tray with 235 seven wells covered with brown paper and four of these containing a mealworm. After this food was restricted for one hour which is a moderate level of deprivation for blue tits and increases their motivation to search for food during the experiment.

The live demonstration was conducted in a plywood cage that was divided into two individual compartments $($ each $66(\mathrm{~h}) \times 50(\mathrm{w}) \times 50(\mathrm{~d}) \mathrm{cm})$ that were separated by a plexiglass wall (Fig. 1b). An individual that was tested was placed on one side of the wall, and a demonstrator bird (or a tray only for the control group) on the other side. Outside the experiment, the plexiglass was covered (with a cardboard sheet), so that the birds could not see each other, and the cover was removed only for the duration of the demonstration. The front wall of each compartment was similarly made of plexiglass, so that we could observe the birds during the experiment. The demonstrator was placed in the test cage two hours before the test (with plexiglass between the two cage compartments covered). Demonstrators were then given one more training session with the symbols to ensure that they were foraging in the test cage, and that they were choosing the 249 right symbol (the symbol they had been trained to associate with a reward). After this, 250 demonstrators were food-deprived for one hour, so that they were motivated to forage during the 
251 demonstration. We then removed the cover of the plexiglass between the observer and the

252 demonstrator, and let the birds to habituate to this new situation for five minutes before presenting

253 the foraging tray to the demonstrator. The tray had three wells covered and one of them (the well

254 with either a cross or a square symbol) contained three mealworms. The order of the symbols was

255 randomized across presentations. We waited until the demonstrator found and ate all three

256 mealworms which took on average 230 seconds (range $=154-492 \mathrm{~s})$.

257

Once the demonstration was finished (i.e. the demonstrator had consumed all three mealworms), we covered again the plexiglass between the cages, so that the birds could not see each other. We then presented observers with a foraging tray with the same three symbols. The order of the symbols in the presented tray was the same as in the demonstration, so that observers could use both symbol and spatial cues about the location of the food reward. This time all the wells were empty to make sure that birds could not get any additional cues about food. We recorded observers' first choice to search for food (i.e. the well where they first lifted up the cover) and the test was finished after this. To investigate whether social information influenced the birds' latency to start the task, we also recorded the elapsed time (s) before the choice. The live control treatment was conducted in a similar way but instead of seeing a demonstrator, birds saw only the tray in an empty cage for 150 seconds.

269

When birds received information from videos, the experiment was conducted in a $66(\mathrm{~h}) \times$ $50(w) \times 50(d)$ cm sized plywood cage with the front wall made of plexiglass. We presented birds

272 videos by placing an LCD monitor (Dell E198FPF, 19", resolution $1280 \times 1024,75 \mathrm{~Hz}$ refresh 273 rate, $300 \mathrm{~cd} / \mathrm{m}^{2}$ ) against the plexiglass (Fig. 1c), following previously validated methods 
274 (Hämäläinen et al., 2017; Thorogood, Kokko \& Mappes, 2018; Hämäläinen et al., 2019). The size

275 of the demonstrator on the screen was smaller than the size of the live bird (approximately $70 \%$

276 of the real size). How birds perceive the demonstrator's size is, however, difficult to estimate

277 because of depth cues (Zeil, 2000) and differences in viewing distance, depending on an observer's

278 position in the cage. Nevertheless, previous studies have demonstrated that great tits use social

279 information from the videos with a similar sized demonstrator (Thorogood, Kokko \& Mappes,

280 2018; Hämäläinen et al., 2019). Birds were first let to habituate to the monitor for 15 min before

281 starting the video. Birds that received social information were then presented a five-minute video

282 of a demonstrator in the cage without the foraging tray, so that the protocol was similar to the live

283 demonstration treatment where birds could observe each other for five minutes before the

284 demonstration. Birds were then presented with a 150 seconds long video of a demonstrator finding

285 and consuming three mealworms under one of the symbols. Birds in the control group saw a video

286 of the feeding tray only (150 s). After this, the computer monitor was removed and we presented

287 birds with the foraging task, following the same protocol as in live demonstration. Again, the order

288 of the symbols was the same as on the videos, and we recorded birds' first choice and the time 289 before they started the task.

290

291 Statistical analyses

292 We first investigated whether birds had an overall preference towards any of the symbols using a

293 binomial test (compared to equal probability of choosing any of the three symbols). We then

294 investigated whether these preferences differed between socially educated and control birds.

295 Because we did not find differences in information use between video and live demonstration

296 treatments (see results), we combined these treatments and used a G-test to compare distributions 
297 of the preferences between all socially educated birds (live and video treatment; $\mathrm{n}=20$ ) and control

298 birds (live and video treatment; $\mathrm{n}=20$ ). We also used a G-test to investigate (i) if birds had a

299 preference for the spatial location on the tray (left/middle/right), i.e. if they chose any of the

300 locations more often than expected by chance (1/3 probability) and to (ii) compare the choices of

301 socially educated birds that saw a demonstrator choosing a square to those seeing a demonstrator

302 choosing a cross (video and live treatments combined). Because birds seemed to prefer a square

303 symbol (see results), we did this by comparing the likelihoods to choose a square (over alternative

304 options cross/white), i.e. testing if birds chose a square more often after seeing a demonstrator

305 choosing it, compared to seeing a demonstrator choosing a cross. We next used a Fisher's exact

306 test to investigate if birds were more likely to copy a demonstrator's choice when they were (i)

307 presented a live demonstrator, compared to video playback, and (ii) when a demonstrator chose a

308 square, compared to a cross. This was done by simply comparing the number of birds whose choice

309 matched that of a demonstrator to those who chose a different symbol. Finally, we tested if social

310 information influenced the latency to start the foraging task using a Cox regression analysis. The

311 time to choose the well (s) was used as a response variable and this was explained by an interaction

312 term between social information treatment (social information/control) and the way information

313 was presented (live/video demonstration). Other explanatory variables in the model included the

314 symbol (cross/square/white) and the tray location (left/middle/right) that the birds chose. To

315 investigate whether birds that matched a demonstrator's choice started the foraging task faster than

316 those that did not, we also conducted the analysis including only socially educated birds (live and

317 video treatment; $n=20$ ). The latency to choose was again used as a response variable and this was

318 explained by an interaction term between information type (live/video demonstrator) and whether 
319 birds chose a same symbol as a demonstrator or not. All the analysis were conducted with the

320 software R.3.3.1 (R Core Team 2016), using survival package (Therneau, 2015).

\section{RESULTS}

323 Overall, birds chose the well with a square symbol more often than predicted by chance (binomial 324 test, 25/40, $\mathrm{p}<0.001)$. This preference, however, differed between socially educated and control 325 birds (G-test, $\mathrm{G}=7.16, \mathrm{p}=0.028$; Fig. $2 \mathrm{a}$ ): individuals that received social information (live and 326 video treatments combined) showed a strong preference towards a square symbol (binomial test, $32715 / 20, p<0.001$ ), whereas this preference was not significant in the control groups (binomial test, $10 / 20, p=0.15)$. Against our prediction that socially educated birds would choose the same symbol as a demonstrator, we did not find evidence that a demonstrator's choice (cross/square) influenced an observers' likelihood to choose a square symbol (G-test, $G=0.51, p=0.47)$. Instead, socially educated birds seemed to prefer a square, regardless of a demonstrator's choice (Fig. 2a). This did not differ between live and video presentations, i.e. birds were not more likely to copy the choice of a live demonstrator compared to video playback (Fisher's exact test, $\mathrm{p}=1$; Fig. 2b). Because demonstrator's choice when a demonstrator chose a square symbol compared to a demonstrator choosing a cross (Fisher's exact test, $\mathrm{p}=0.003$ ). The location on the tray (left $/ \mathrm{middle} / \mathrm{right}) \mathrm{did}$ not influence birds' choices (location that birds chose did not differ from that expected by random chance; G-test, $\mathrm{G}=3.62, \mathrm{p}=0.16)$. demonstration, compared to live demonstration groups (effect of video presentation: coefficient $=$ 
$3421.072 \pm 0.420, Z=2.553, p=0.01)$. Birds that chose the right side of the tray also initiated the task

343 faster than birds that chose the left side (effect of location (right): coefficient $=1.086 \pm 0.458, Z=$

$3442.372, p=0.02)$. Birds tended to choose a square symbol faster than a cross symbol, but this effect

345 was marginal (effect of symbol (square): coefficient $=0.918 \pm 0.526, \mathrm{Z}=1.745, \mathrm{p}=0.08$ ).

346 Received social information did not influence how fast birds started to forage (effect of social

347 information: coefficient $=-0.210 \pm 0.382, Z=-0.549, p=0.58)$, regardless of the way the

348 information was presented (social information * type of presentation (video): coefficient $=0.265$

$349 \pm 0.726, Z=0.366, p=0.71)$, and these non-significant terms were removed from the final model.

350 However, when investigating only socially educated birds, we found that birds that matched a

351 demonstrator's choice started the foraging task more quickly (mean $=81 \mathrm{~s}$, range $=12-253 \mathrm{~s})$ than

352 those that did not $($ mean $=768 \mathrm{~s}$, range $=35-2640 \mathrm{~s}$; matching a demonstrator: coefficient $=1.058$

$353 \pm 0.539, \mathrm{Z}=1.962, \mathrm{p}=0.049)$. This did not depend on the way information was presented

354 (matching a demonstrator * type of presentation (video): coefficient $=-0.635 \pm 0.970, Z=-0.655$,

$355 \mathrm{p}=0.51$ ), and this interaction was excluded from the final model.

356

357 DISCUSSION

358 In this experiment, we tested whether blue tits were more likely to copy the food choice of a live 359 conspecific, compared to video playback. However, we found that blue tits did not copy a 360 demonstrator's choice of symbol, regardless of how social information was presented. Instead, 361 individuals chose the well with a square symbol more often than other options (Fig. 2a). Because 362 of this preference and the lack of evidence that observers copied a demonstrator's choice, it is 363 difficult to compare the effectiveness of video playback and live demonstration. However, birds'

364 preference for a square symbol was stronger after they received social information, compared to 
365 the control groups, and birds whose choice matched that of their demonstrator were quicker to

366 initiate foraging. These responses were consistent across both social information treatments,

367 indicating that even if birds did not often choose the same symbol as a demonstrator, they

368 responded to video and live presentations similarly.

Blue tits might not value social information when the foraging task is relatively simple.

371

372

373

374

375

376

377

378

379

380

381

382

383

384

385

386

387

Similar to our previous video playback study (Hämäläinen et al., 2017), we did not find evidence that blue tits copied the foraging choice of a conspecific from the video, and neither did they copy the choice of a live demonstrator. Other studies with live demonstrators have similarly failed to find a strong effect of social information in blue tits, showing that only approximately $50 \%$ of tested birds learn a novel foraging task socially (Sasvári, 1979; Sasvári, 1985; Aplin, Sheldon \& Morand-Ferron, 2013). Social learning seems to be also age- and sex-biased with juveniles (Sasvári 1985) and especially juvenile females being more likely to learn socially (Aplin, Sheldon \& Morand-Ferron, 2013). To increase the chances to detect social information use, we therefore decided to test only juveniles, but we were not able to determine the sex of the tested individuals. Furthermore, birds were provided with both visual and spatial cues about the food reward (the location of the symbols in the foraging task mirrored that in the demonstration), so individuals could have used either type of information. Despite this, we failed to find evidence of blue tits copying a demonstrator's foraging choice. However, similar to our previous study (Hämäläinen et al., 2017), we found that birds that matched a demonstrator's choice started the foraging task more quickly than birds that chose an alternative symbol, suggesting that social information did influence their behaviour. In addition, birds started the task faster after seeing video playback (either control or social information) compared to seeing live stimuli. This probably results from 
388 slight differences between the test conditions (i.e. different test cages). After the live

389 demonstration, we covered the observer's view of the demonstrator's cage by sliding a cardboard

390 sheet between the two cage compartments, and this disturbance might have affected the observers

391 more than simply removing the computer monitor following the video demonstration. Therefore,

392 the test with live stimuli might have been slightly more stressful for the birds which could explain

393 the longer hesitation to start the foraging task.

394

Despite failing to find evidence that blue tits copied the foraging choice of a demonstrator, 396 social information did have an influence on their foraging choices. In all treatments, birds chose the square symbol more often than other two options (cross or white). However, this preference for squares was even stronger when birds received social information from a live or video demonstrator, regardless of the demonstrator's choice. This indicates that simply seeing a demonstrator foraging from the tray enhanced blue tits' preference towards the square symbol. This result is difficult to explain, but it is possible that birds saw a demonstrator as a competitor, which made them to choose the most visible and preferred prey item. Blue tits were similarly found to prefer squares in another experiment, where birds were allowed to choose between two prey items with cross and square symbols (Hämäläinen et al., in review). A conspicuous square therefore seems to be a more salient cue for blue tits, and contrasting social information about food location did not override this preference. Great tits were recently found to have a high level of selfcontrol ability (Isaksson, Urhan \& Brodin, 2018), but to our knowledge this has not been tested in blue tits, and it is possible that blue tits were simply too impulsive to inhibit their response to the preferred signal. This initial preference makes our results difficult to interpret, and different 410 symbol choice might have provided us better evidence of social information use. Interestingly, the 
411 preference for square symbols has not been found in great tits (Lindström et al., 2001; Hämäläinen

412 et al., 2019), and artificial prey with cross and square symbols have been used in many avoidance

413 learning experiments (e.g. Alatalo \& Mappes, 1996; Lindström et al., 1999; Lindström et al., 2001;

414 Thorogood, Kokko \& Mappes, 2018). In these experiments squares often represent unpalatable

415 aposematic prey and great tits acquire avoidance to squares faster after receiving social information

416 about their unpalatability (Thorogood, Kokko \& Mappes, 2018; Hämäläinen et al., 2019). Despite

417 the initial preference for squares, blue tits similarly learn to avoid them faster after observing a

418 negative foraging experience of a conspecific (Hämäläinen et al., in review) which shows that blue

419 tits can switch their foraging preferences according to acquired social information. However, our

420 experiment suggests that this is context-dependent, and blue tits do not change their preferences

421 when they receive positive social information and the foraging task is relatively simple.

422

Our study highlights the importance of comparing animals' response to real and video 424 stimuli when testing the applicability of video playback (D'Eath, 1998). Without the live demonstrator treatment, it would have been difficult to separate the effect of video presentation

426 from blue tits' tendency to use social information. However, because birds were not more likely

427 to copy the choices of live demonstrators, we can now be more confident that our result is not 428 explained only by the lack of response to video playback. Comparing individuals' responses 429 between video and live demonstrations is important even when videos are found to have an effect 430 on observers' behaviour, as these responses could be different compared to live stimuli. The 431 responses to videos might also be context-dependent: zebra finch males showed a stronger 432 preference for the nest material choice of a live conspecific (Guillette \& Healy, 2019), whereas 433 female zebra finches courted video images of males more actively than live males, possibly 
434 because of the lack of reciprocal response from males on the video (Swaddle, McBride \&

435 Malhotra, 2006). The efficacy of video playback seems to also depend on the features of the video

436 presentation, such as the sound on the video. Zebra finches were shown to copy foraging choices

437 from video playback only when videos did not have sound (Guillette \& Healy, 2017), whereas the

438 opposite was true in Burmese red junglefowl (Gallus gallus spadecius) that used social information

439 only from videos that included sound (McQuoid \& Galef, 1993). Together, these studies indicate

440 that video playback can be a useful tool in behavioural studies but its applicability might vary

441 among species and different contexts.

\section{Conclusion}

444 The aim of our study was to test the effectiveness of video playback in social learning studies in blue tits by comparing social information use between live and video demonstrations. This comparison proved to be difficult, as we did not find strong evidence of social learning from either live or video demonstrators, indicating that blue tits do not rely on social information in simple foraging tasks. In our experiment the cost to search for food (i.e. lift up the paper cover) was probably low and birds might have ignored social information because personal information was easy to acquire (Laland, 2004; Kendal et al., 2005). It is also possible that birds would have needed to observe several demonstrations from different individuals before relying on social information. In our experiment individuals received information from one demonstrator only, whereas in nature blue tits form foraging flocks and have opportunities to gather information from both conspecifics and heterospecifics (Farine et al., 2015). Individuals are also likely to vary in their tendency to use social information (Sasvári, 1979; Aplin, Sheldon \& Morand-Ferron, 2013) and we might have 
457 information about the location of food, some observers might have seen the demonstrator as a

458 competitor and therefore avoided the same symbol. Nevertheless, we found that blue tits responded

459 to video playback similarly to a live demonstrator, as both demonstrations enhanced observers'

460 preference towards squares, indicating that videos had the same effect on birds' behaviour as live

461 demonstrators. However, because of the difficulties to detect social learning in blue tits, the

462 efficacy of videos should be tested in other contexts before making conclusions of its applicability 463 for this species.

464

\section{ACKNOWLEDGEMENTS}

466

467

468

469

470

471

472

473

474

475

476

477

478

We thank Helinä Nisu and staff at the Konnevesi Research Station for taking care of the birds and

providing facilities for the experiments, Victoria Franks for providing a blue tit illustration and Lucy Aplin for discussions on the methodology. The manuscript was improved by comments from Patrice Adret and the anonymous referee, as well as three anonymous reviewers at Peerage of Science.

\section{REFERENCES}

Adret, P. (1997). Discrimination of video images by zebra finches (Taeniopygia guttata): Direct evidence from song performance. Journal of Comparative Psychology, 111, 115-125. http://dx.doi.org/10.1037/0735-7036.111.2.115

Alatalo, R.V., \& Mappes, J. (1996). Tracking the evolution of warning signals. Nature, 382, 708-710. https://doi.org/10.1038/382708a0

Aplin, L.M. (2019). Culture and cultural evolution in birds: a review of the evidence. Animal 
480

481

482

483

484

485

486

487

488

489

490

491

492

493

494

495

496

497

498

499

Aplin, L.M., Sheldon, B.C., \& Morand-Ferron, J. (2013). Milk bottles revisited: social learning and individual variation in the blue tit, Cyanistes caeruleus. Animal Behaviour, 85, 12251232. https://doi.org/10.1016/j.anbehav.2013.03.009

Baracchi, D., Vasas, V., Jamshed Iqbal, S., \& Alem, S. (2018). Foraging bumblebees use social cues more when the task is difficult. Behavioral Ecology, 29, 186-192. https://doi.org/10.1093/beheco/arx143

Bird, C.D., \& Emery, N.J. (2008). Using video playback to investigate the social preferences of rooks, Corvus frugilegus. Animal Behaviour, 76, 679-687. http://dx.doi.org/10.1016/j.anbehav.2008.04.014

Boström, J.E., Dimitrova, M., Canton, C., Håstad, O., Qvarnström, A., \& Ödeen, A. (2016). Ultra-rapid vision in birds. PLoS One, 11, e0151099. https://doi.org/10.1371/journal.pone.0151099

Brecht, K.F., Ostojić, L., Legg, E.W., \& Clayton, N.S. (2018). Difficulties when using video playback to investigate social cognition in California scrub-jays (Aphelocoma californica). PeerJ, 6, e4451. https://doi.org/10.7717/peerj.4451

Brown, C., \& Laland, K.N. (2003). Social learning in fishes: A review. Fish and Fisheries, 4, 280-288. https://doi.org/10.1046/j.1467-2979.2003.00122.x

Carouso-Peck, S., \& Goldstein, M.H. (2019). Female social feedback reveals non-imitative mechanisms of vocal learning in zebra finches. Current Biology, 29, 631-636. https://doi.org/10.1016/j.cub.2018.12.026 
500 Clark, D. L., Macedonia, J.M., \& Rosenthal, G.G. (1997). Testing video playback to lizards in 501 the field. Copeia, 2, 421-423. https://doi.org/10.2307/1447764

502 Clark, D.L., \& Uetz, G.W. (1992). Morph-independent mate selection in a dimorphic jumping 503 spider: demonstration of movement bias in female choice using video-controlled courtship 504 behaviour. Animal Behaviour, 43, 247-254. https://doi.org/10.1016/S0003-3472(05)802205059

506

507

508

509

510

511

512

513

514

515

516

517

518

519

D’Eath, R.B. (1998). Can video images imitate real stimuli in animal behaviour experiments? Biological Reviews, 73, 267-292. http://doi.org/10.1111/j.1469-185X.1998.tb00031.x

Danchin, E., Giraldeau, L.A., Valone, T.J., \& Wagner, R.H. (2004). Public information: from nosy neighbors to cultural evolution. Science, 305, 487-491. https://doi.org/10.1126/science.1098254

Dawson, E.H., \& Chittka, L. (2012). Conspecific and heterospecific information use in bumblebees. PLoS One, 7, e31444. https://doi.org/10.1371/journal.pone.0031444

Farine, D.R., Aplin, L.M., Sheldon, B.C., \& Hoppitt, W. (2015). Interspecific social networks promote information transmission in wild songbirds. Proceedings of the Royal Society B: Biological Sciences, 282, 20142804. https://doi.org/10.1098/rspb.2014.2804

Galef Jr, B.G., \& Giraldeau, L.A. (2001). Social influences on foraging in vertebrates: causal mechanisms and adaptive functions. Animal Behaviour, 61, 3-15. https://doi.org/10.1006/anbe.2000.1557

Galef, B.G., \& Laland, K.N. (2005). Social learning in animals: empirical studies and theoretical 
520

521

522 523

524

525

526

527

528

529

530

531

532

533

534

535

536

537

538

539

540

models. Bioscience, 55, 489-499. https://doi.org/10.1641/00063568(2005)055[0489:SLIAES]2.0.CO;2

Guillette, L.M., \& Healy, S.D. (2017). The roles of vocal and visual interactions in social learning zebra finches: a video playback experiment. Behavioural Processes, 139, 43-49. https://doi.org/10.1016/j.beproc.2016.12.009

Guillette, L.M., \& Healy, S.D. (2019). Social learning in nest-building birds watching livestreaming video demonstrators. Integrative Zoology. 14, 204-213. https://doi.org/10.1111/1749-4877.12316

Gunhold, T., Whiten, A., \& Bugnyar, T. (2014). Video demonstrations seed alternative problemsolving techniques in wild common marmosets. Biology Letters, 10, 1-5. http://dx.doi.org/10.1098/rsbl.2014.0439

Halnes, S.E., \& Gould, J.L. (1994). Female platys prefer long tails. Nature, 370, 512. https://doi.org/10.1038/370512a0

Hodgson, Z.G., \& Healy, S.D. (2005). Preference for spatial cues in a non-storing songbird species. Animal Cognition, 8, 211-214. https://doi.org/10.1007/s10071-004-0249-4

Hopper, L.M., Lambeth, S.P., \& Schapiro, S.J. (2012). An evaluation of the efficacy of video displays for use with chimpanzees (Pan troglodytes). American Journal of Primatology, 74, 442-449. https://doi.org/10.1002/ajp.22001

Hämäläinen, L., Mappes, J., Rowland, H.M., \& Thorogood, R. (2019). Social information use about novel aposematic prey is not influenced by a predator's previous experience with toxins. Functional Ecology. https://doi.org/10.1111/1365-2435.13395 
541 Hämäläinen, L., Rowland, H.M., Mappes, J., \& Thorogood, R. (2017). Can video playback

542 provide social information for foraging blue tits?. PeerJ, 5, e3062.

$543 \quad$ https://doi.org/10.7717/peerj.3062

544 Höglund, J., Alatalo, R.V., Gibson, R.M., \& Lundberg, A. (1995). Mate-choice copying in black 545 grouse. Animal Behaviour, 49, 1627-1633. https://doi.org/10.1016/0003-3472(95)90085-3

546 Ikebuchi, M., \& Okanoya, K. (1999). Male zebra finches and Bengalese finches emit directed 547 songs to the video images of conspecific females projected onto a TFT display. Zoological $548 \quad$ Science, 16, 63-71. http://doi.org/10.2108/zsj.16.63

549 Isaksson, E., Utku , A.U., \& Brodin, A. (2018). High level of self-control ability in a small $550 \quad$ passerine bird. Behavioral Ecology and Sociobiology, 72, 118.

$551 \quad$ https://doi.org/10.1007/s00265-018-2529-z

552 Kendal, R.L., Boogert, N.J., Rendell, L., Laland, K.N., Webster, M., \& Jones, P.L. (2018). Social 553 learning strategies: Bridge-building between fields. Trends in Cognitive Sciences. 22, 651554 665. https://doi.org/10.1016/j.tics.2018.04.003

555 Kendal, R. L., Coolen, I., van Bergen, Y., \& Laland, K.N. (2005). Trade-offs in the adaptive use 556 of social and asocial learning. Advances in the Study of Behavior, 35, 333-379.

$557 \quad$ http://dx.doi.org/10.1016/S0065-3454(05)35008-X

558 Kis, A., Huber, L., \& Wilkinson, A. (2015). Social learning by imitation in a reptile (Pogona 559 vitticeps). Animal Cognition, 18, 325-331. https://doi.org/10.1007/s10071-014-0803-7

560 Krause, J., Winfield, A.F.T., \& Deneubourg, J.L. (2011). Interactive robots in experimental $561 \quad$ biology. Trends in Ecology and Evolution, 26, 369-375. 
Laland, K.N. (2004). Social learning strategies. Learning \& Behavior, 32, 4-14.

564 https://doi.org/10.3758/BF03196002

565

566

567

568

569

570

571

572

573

574

575

576

577

578

579

580

581

582

Lindström, L., Alatalo, R.V., Lyytinen, A., \& Mappes, J. (2001). Predator experience on cryptic prey affects the survival of conspicuous aposematic prey. Proceedings of the Royal Society of London. Series B: Biological Sciences, 268, 357-361. https://doi.org/10.1098/rspb.2000.1377

Lindström, L., Alatalo, R.V., Mappes, J., Riipi, M., \& Vertainen, L. (1999). Can aposematic signals evolve by gradual change?. Nature, 397, 249-251. https://doi.org/10.1038/16692

McQuoid, L.M., \& Galef, B.G. (1993). Social stimuli influencing feeding behaviour of Burmese fowl: video analysis. Animal Behaviour, 46, 13-22. http://dx.doi.org/10.1006/anbe.1993.1157

Noble, D.W.A., Byrne, R.W., \& Whiting, M.J. (2014). Age-dependent social learning in a lizard. Biology Letters, 10, 20140430. https://doi.org/10.1098/rsbl.2014.0430

Ord, T.J., Peters, R.A., Evans, C.S., \& Taylor, A.J. (2002). Digital video playback and visual communication in lizards. Animal Behaviour, 63, 879-890. http://dx.doi.org/10.1006/anbe.2001.1983

Ophir, A.G., \& Galef, B.G. (2003). Female Japanese quail affiliate with live males that they have seen mate on video. Animal Behaviour, 66, 369-375. http://dx.doi.org/10.1006/anbe.2003.2229

Partan, S.R., Larco, C.P., \& Owens, M.J. (2009). Wild tree squirrels respond with multisensory 
583

584

585

586

587

588

589

590

591

592

593

594

595

596

597

598

599

600

601

enhancement to conspecific robot alarm behaviour. Animal Behaviour, 77, 1127-1135. https://doi.org/10.1016/j.anbehav.2008.12.029

Patricelli, G.L., Uy, J.A.C., Walsh, G., \& Borgia, G. (2002). Sexual selection: male displays adjusted to female's response. Nature, 415, 279-280. https://doi.org/10.1038/415279a

Petersson, E., \& Järvi, T. (2006). Anti-predator response in wild and sea-ranched brown trout and their crosses. Aquaculture, 253, 218-228.

https://doi.org/10.1016/j.aquaculture.2005.08.012

Powell, G.V.N. (1974). Experimental analysis of the social value of flocking by starlings (Sturnus vulgaris) in relation to predation and foraging. Animal Behaviour, 22, 501-505. https://doi.org/10.1016/S0003-3472(74)80049-7

R Core Team (2016). R: A language and environment for statistical computing. R Foundation for Statistical Computing, Vienna, Austria. https://www.R-project.org

Rieucau, G., \& Giraldeau, L.A. (2009). Video playback and social foraging: simulated companions produce the group size effect in nutmeg mannikins. Animal Behaviour, 78, 961-966.

http://dx.doi.org/10.1016/j.anbehav.2009.06.023

Roberts, N.S., Gumm, J.M., \& Mendelson, T.C. (2017). Darter (Percidae: Etheostoma) species differ in their response to video stimuli. Animal Behaviour, 131, 107-114. https://doi.org/10.1016/j.anbehav.2017.07.013 
602 Rowland, W.J., Bolyard, K.J., Jenkins, J.J., \& Fowler, J. (1995). Video playback experiments on 603 stickleback mate choice: female motivation and attentiveness to male colour cues. Animal 604 Behaviour, 49, 1559-1567. http://dx.doi.org/10.1016/0003-3472(95)90077-2

605 Sasvári, L. (1979). Observational learning in great, blue and marsh tits. Animal Behaviour, 27, 606 767-771. https://doi.org/10.1016/0003-3472(79)90012-5

607 Sasvári, L. (1985). Different observational learning capacity in juvenile and adult individuals of 608 congeneric bird species. Zeitschrift für Tierpsychologie, 69, 293-304.

609 https://doi.org/10.1111/j.1439-0310.1985.tb00154.x

610

611

612

613

614

615

616

617

618

619

620

621

622

Schlupp, I., 2000. Are there lessons from negative results in studies using video playback? Acta Ethologica, 3, 9-13. https://doi.org/10.1007/s102110000026

Smit, J.A., \& van Oers, K. (2019). Personality types vary in their personal and social information use. Animal Behaviour. 151, 185-193. https://doi.org/10.1016/j.anbehav.2019.02.002

Snijders, L., Naguib, M., \& van Oers, K. (2017). Dominance rank and boldness predict social attraction in great tits. Behavioral Ecology, 28, 398-406. https://doi.org/10.1093/beheco/arw158

Swaddle, J.P., McBride, L., \& Malhotra, S. (2006). Female zebra finches prefer unfamiliar males but not when watching noninteractive video. Animal Behaviour, 72, 161-167. https://doi.org/10.1016/j.anbehav.2005.12.005

Taylor, R.C., Klein, B.A., Stein, J., \& Ryan, M.J. (2008). Faux frogs: multimodal signalling and the value of robotics in animal behaviour. Animal Behaviour, 76, 1089-1097. https://doi.org/10.1016/j.anbehav.2008.01.031 
623 Therneau, T. (2015). A Package for Survival Analysis in S. version 2.38. https://CRAN.R-

624 project.org/package $=$ survival

625 Thorogood, R., \& Davies, N.B. (2016). Combining personal with social information facilitates

626 host defences and explains why cuckoos should be secretive. Scientific Reports, 6, 19872.

627 https://doi.org/10.1038/srep19872

628 Thorogood, R., Kokko, H., \& Mappes, J. (2018). Social transmission of avoidance among 629 predators facilitates the spread of novel prey. Nature Ecology \& Evolution, 2, 254-261. 630 https://doi.org/10.1038/s41559-017-0418-x

631 Tinbergen, N., \& Perdeck, A.C.C. (1950). On the stimulus situation releasing the begging 632 response in the newly hatched herring gull chick (Larus a. argentatus Pont.). Behaviour, 3, $633 \quad 1-39$.

634 Trainor, B.C., \& Basolo, A.L. (2000). An evaluation of video playback using Xiphophorus 635 helleri. Animal Behaviour, 59, 83-89. https://doi.org/10.1006/anbe.1999.1289

636 Vallortigara, G., \& Regolin, L. (2006). Gravity bias in the interpretation of biological motion by 637 inexperienced chicks. Current Biology, 16, R279-R280.

638 https://doi.org/10.1016/j.cub.2006.03.052

639 Vallortigara, G., Regolin, L., \& Marconato, F. (2005). Visually inexperienced chicks exhibit 640 spontaneous preference for biological motion patterns. PLoS biology, 3, e208.

641 https://doi.org/10.1371/journal.pbio.0030208 
642 Ware, E., Saunders, D.R., \& Troje, N.F. (2015). The influence of motion quality on responses

643 towards video playback stimuli. Biology Open, 4, 803-811.

644 https://doi.org/10.1242/bio.011270

645 Whiten, A. (2000). Primate culture and social learning. Cognitive Science, 24, 477-508.

$646 \quad$ https://doi.org/10.1207/s15516709cog2403_6

647 Woo, K.L., \& Rieucau, G. (2011). From dummies to animations: A review of computer-

648 animated stimuli used in animal behavior studies. Behavioral Ecology and Sociobiology, 65,

649 1671-1685. http://dx.doi.org/10.1007/s00265-011-1226-y

650 Zeil, J. (2000). Depth cues, behavioural context, and natural illumination: some potential

651 limitations of video playback techniques. Acta Ethologica, 3, 39-48.

652 https://doi.org/10.1007/s102110000021

653

654 


\section{Figure 1}

The experimental set-up.

(A) An example of the ice cube tray that was presented to birds. The tray had 21 wells and three of them (left, middle and right well in the middle row) were covered with a piece of white paper that had either a black cross or a square printed on top, or no symbols (plain white). The same symbols were attached in front of the tray to increase their visibility to observers. The order of the symbols was randomized among birds. (B) The set-up of the live demonstration. The demonstrator (left) and the observer (right) were in individual cages that were separated by plexiglass, so that birds could see each other. In the control treatment the birds saw only the tray. (C) The set-up of the video playback. A computer monitor was placed against a plexiglass front wall of the test cage. Birds were then presented a video of a demonstrator or a control video of the tray. Blue tit illustration credit: Victoria Franks 
a)

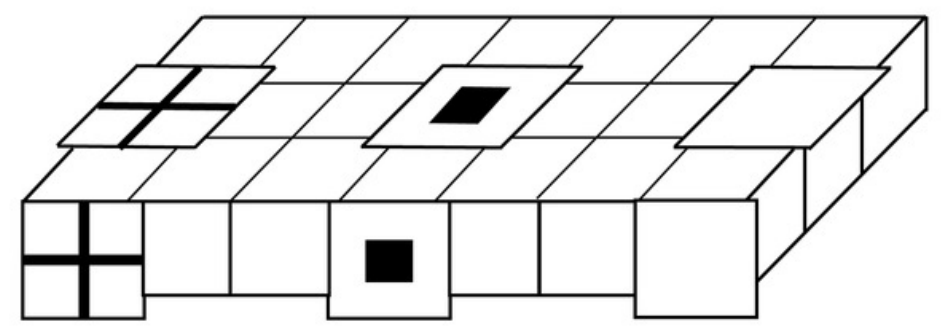

b)

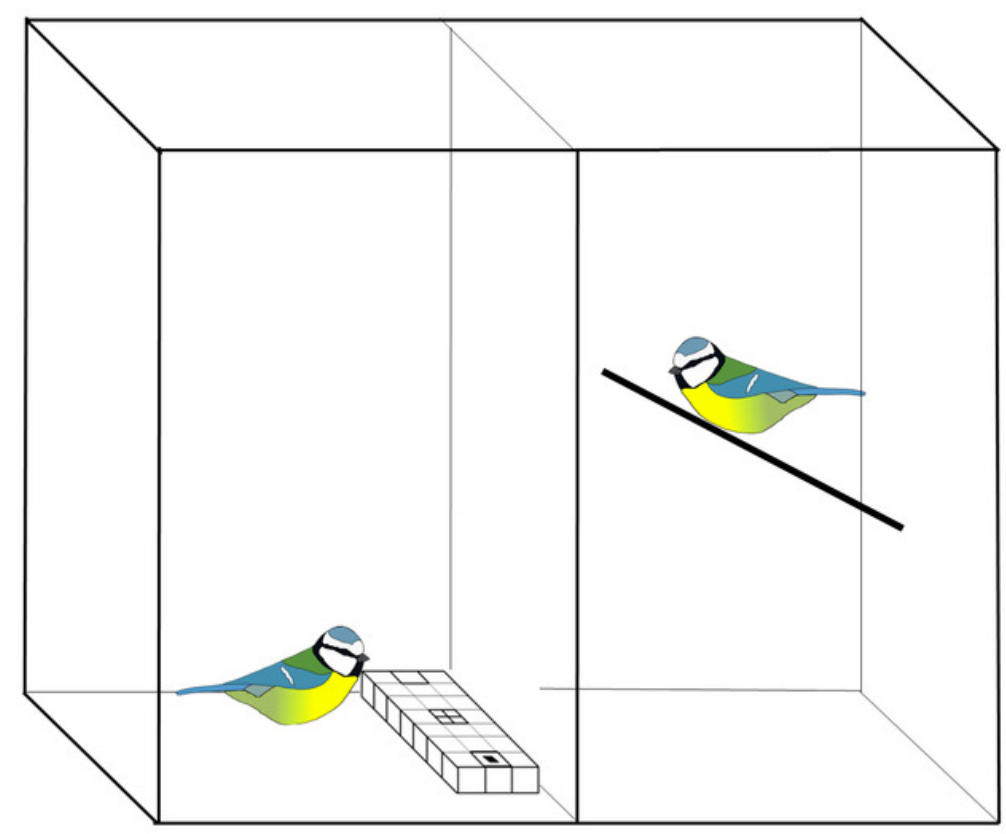

c)

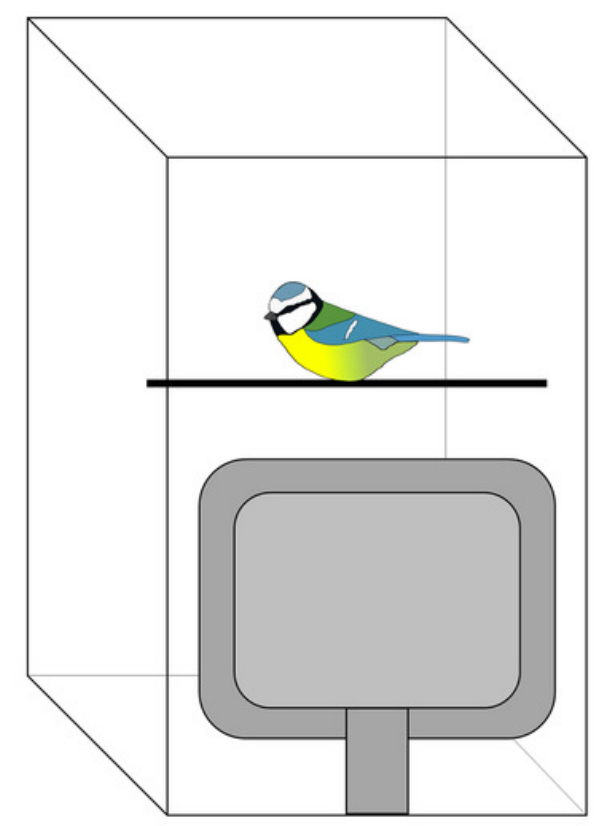




\section{Figure 2}

Birds' foraging choices in the experiment.

(A) The percentage of birds $(n=40)$ choosing each symbol when they were presented (live and video demonstrations combined) (i) a tray only (light grey bars, $n=20$ ), (ii) social information of a demonstrator choosing a cross (dark grey bars, $n=10$ ), or (iii) social information of a demonstrator choosing a square (black bars, $n=10$ ). In the absence of any symbol preference by the birds, each symbol was predicted to be chosen with 1/3 probability. This is represented by the dotted line (33\%) and the bars above the line indicate birds' preference towards that symbol. (B) Percentage of socially educated birds $(n=20)$ that copied the demonstrator (i.e. chose the same symbol as a demonstrator vs. one of the other two symbols) after seeing a live demonstrator (light grey bars, $\mathrm{n}=10$ ) or video playback of a demonstrator (dark grey bars, $\mathrm{n}=10$ ). 

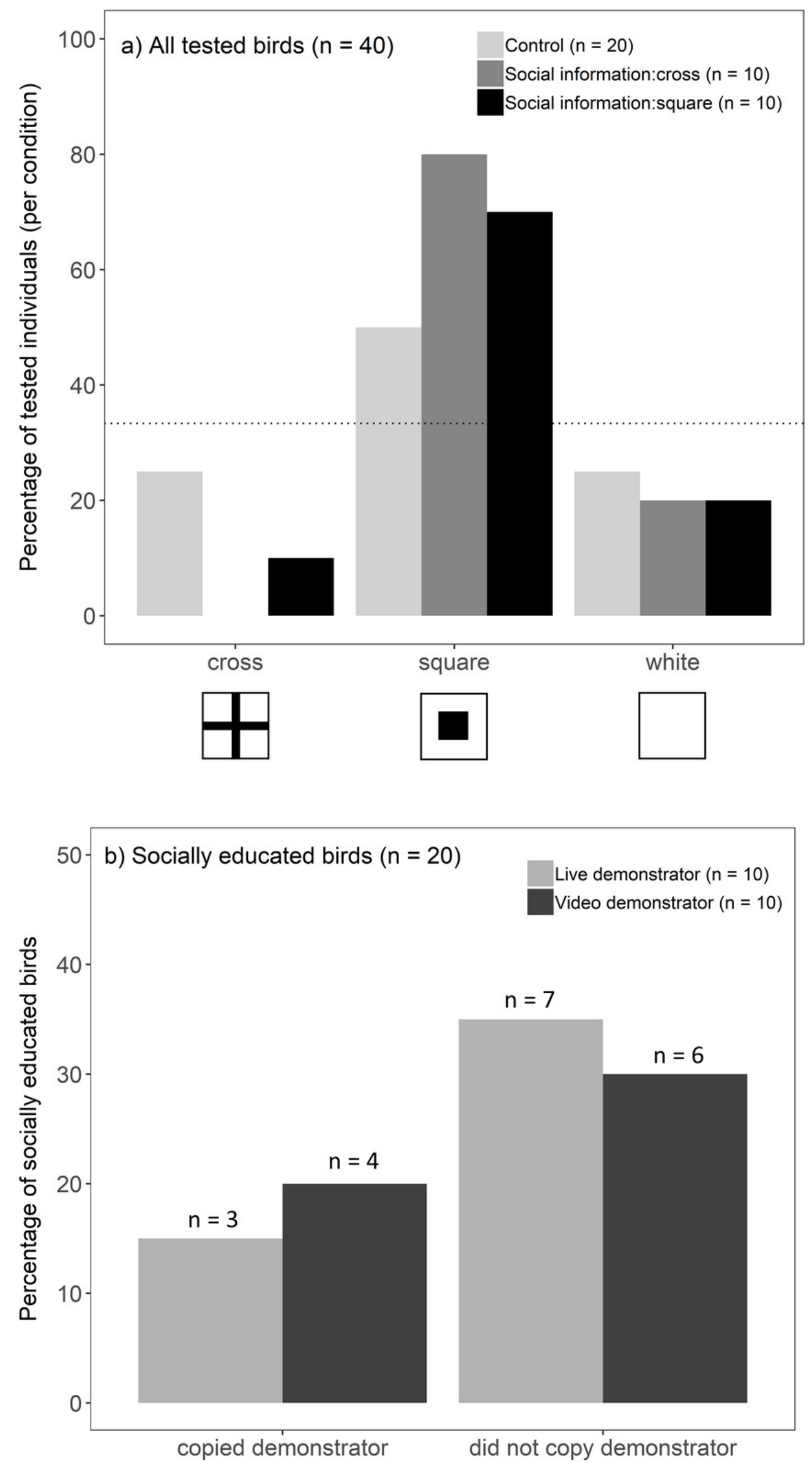